\title{
Laparoscopic Sacrocolpopexy Mesh Removal for Persistent Lumbosacral and Right-sided Gluteal Pain Possibly Caused by Nerve "entrapment"
}

\section{Li Yan}

Chongqing Health Center for Women and Children

\section{Lu Bin Liu}

Chongqing Health Center for Women and Children

Cheng Zhi Zhao ( $\sim$ Zhaochengzhi952@163.com )

Chongqing Health Center for Women and Children

\section{Case report}

Keywords: Laparoscopic sacrocolpopexy, Chronic pelvic pain, Mesh complications, Mesh excision

Posted Date: May 28th, 2021

DOI: https://doi.org/10.21203/rs.3.rs-543673/v1

License: (c) (i) This work is licensed under a Creative Commons Attribution 4.0 International License.

Read Full License 


\section{Abstract}

Background: Laparoscopic sacrocolpopexy (LSC), which is an important procedure for vault prolapse, has the advantages of high cure rate, low recurrence rate and little impact on sexual life. Few nerve entrapment complications have been reported in the literature.

Case presentation: A 48-year-old woman developed persistent lumbosacral and right-sided gluteal pain after the LSC. During diagnostic laparoscopy, we found that the fixation site deviated by approximately $1.5 \mathrm{~cm}$ from the middle of the sacral promontory, the anterior surface of the first sacral vertebra. Hence we removed most of the mesh carefully. S1 nerve "entrapment" caused by this deviation, accompanied by the tissue fibrosis and scar tissue formation, may have been the main cause of pain. The patient's symptoms resolved after surgical intervention.

Conclusions: Our case emphasizes that sacral nerve entrapment is a potential complication of retroperitonealized synthetic mesh placement during sacrocolpopexy. We hope that this report can serve as a reference for dealing with similar situations. For skilled laparoscopists, laparoscopic mesh removal is safe and feasible, but attention should be paid to avoid damage to vessels, ureters and other important organs.

\section{Background}

Prolapse of the vaginal vault is a common complication after hysterectomy[1]. It may occur when structures that stabilise the apex of the vagina are not restored at surgery or when these structures dilate[2]. Although the exact incidence of post-hysterectomy prolapse is unknown, it is estimated to be 6$8 \%[1]$. Laparoscopic sacrocolpopexy (LSC), which is an important procedure for vault prolapse, has the advantages of high cure rate, low recurrence rate and little impact on sexual life. Few nerve entrapment complications have been reported in the literature.

\section{Case Presentation}

A 48-year-old woman was referred to our unit; she underwent laparoscopic sacrocolpopexy (LSC) with a Y-shaped mesh (ARTISYN; Johnson \& Johnson international, c/o European Logistics Centre, Diegem, Belgium) for vault prolapse after having undergone hysterectomy for cervical intraepithelial neoplasia III (CINIII) 6 months earlier. She began to feel pain in the lumbosacral region approximately 3 weeks after the surgery, and then, she developed right-sided gluteal pain, which was persistent and gradually worsened.

The clinical examination revealed that mesh erosion was not present in the vagina or vaginal vault, but there was a point of local tenderness, which measured $1.5 \mathrm{~cm} \times 1.5 \mathrm{~cm}$ and was located near the ischial tubercle of the right hip. No sensory function, motor function or reflex abnormalities were detected by neurological examinations of either of the lower limbs or lumbosacral region. She did not have any bowel or bladder symptoms. The serum white blood cell (WBC) count, C-reactive protein (CRP) and plasma 
procalcitonin (PCT) levels were in the normal range. Magnetic resonance imaging of the pelvis showed that the size of encapsulated pelvic effusion was $5 \star 3.5 \star 4.6 \mathrm{~cm}$, with an irregular shape accompanying mesh echo from the top of the vagina to the presacral area (Fig. 1A B C), but the specific fixed point could not be clearly identified. We didn't treat the patient with antibiotics since there were no signs of inflammation. The patient was repeatedly treated for pain by various means, including acupuncture, electromagnetic therapy, and analgesics, and psychological experts were invited to participate in the treatment and follow-up sessions for the patient; however, none of these methods had obvious effects. Therefore, she was admitted to the hospital for treatment at the last outpatient follow-up. After admission, we held a multidisciplinary consultation and decided to carry out laparoscopic exploration and finally remove the mesh.

The patient received one dose of cefazolin, a first-generation cephalosporin, pre-operatively. During laparoscopy, we found that adhesions were located between the sigmoid colon and the apex of the vagina, secondary to the mesh in the peritoneal cavity. We separated the adhesions gradually and carefully, and then, we found tension and wrinkles in the mesh. The surface of the mesh was covered with a thin layer of peritoneum (Fig. 2A). When separated the adhesions, we found the nonabsorbable sutures from the last operation (Fig. 2B) and encapsulated effusion of the right pelvis (Fig. 2A B), which was consistent with the magnetic resonance imaging findings. We could also see that the appendix adhered to the fixation site of the mesh (Fig. 2B). We didn't remove the appendix since there were no signs of inflammation or abscess. We dissected the right internal iliac vessels and ureters to better visualize the surgical field and to avoid damage to important tissues and organs (Fig. 3A). We found that compared with the fixation sites of our routine operations, the mesh's fixation site in this patient deviated toward the right side of the sacrum and closer to the internal iliac vessels; the site deviated by approximately $1.5 \mathrm{~cm}$ from the middle of the sacral promontory, the anterior surface of the first sacral vertebra (S1) (Fig. 3A B). We performed bacterial cultures of the retention fluid and the mesh removed from the surgery. The reports were negative.

\section{Discussion}

In this case, $\mathrm{S} 1$ nerve "entrapment" caused by this deviation, accompanied by the tissue fibrosis and scar tissue formation, may have been the main cause of pain. Because the S1 nerve was positioned approximately $2.5 \mathrm{~cm}$ below the midpoint of the sacral promontory and $2 \mathrm{~cm}$ to the right of the midline[3], the site of the suture and fixation deviated toward the right side of the sacrum, which increased the risk of S1 nerve injury. Other causes of the pain should be considered, including excessive tension and wrinkling of the mesh[4], adhesion of the mesh to the appendix[5], the formation of encapsulated effusion and persistent potential infection on the mesh.

There was complete resolution of her symptoms without the need for any neuropathic pain medications or opioids at the 6-month follow-up in the hospital. But there was a minimal vault descent, the state of which is needed to be followed up long term. 
It was difficult to determine whether to perform laparoscopic exploration and remove the mesh before surgery because few similar cases have been reported[6]. The incidence of nerve entrapment after LSC with mesh is unclear, and the nerve injury incidence has been underestimated[7]; thus, the risks associated with the use of mesh in the treatment of pelvic organ prolapse and stress incontinence should be well documented. Some scholars have tried to perform LSC without mesh, which has some effects, but relevant data are limited, and randomized multicenter studies are needed to draw definitive conclusions[8].

\section{Conclusion}

Our case emphasizes that sacral nerve entrapment is a potential complication of retroperitonealized synthetic mesh placement during sacrocolpopexy. We hope that this report can serve as a reference for dealing with similar situations. Laparoscopy has advantages over the open approach, with good access and visualisation of the entire course of the mesh, and more rapid recovery for the patient. For skilled laparoscopists, laparoscopic mesh removal is safe and feasible, but attention should be paid to avoid damage to vessels, ureters and other important organs.

\section{Declarations}

\section{Acknowledgements}

Not applicable.

\section{Author contributions}

Chengzhi Zhao and Lubin Liu completed the operation. Li Yan and Chengzhi Zhao were in charge of the patient. Li Yan compiled the medical records and wrote the manuscript. All authors read and approved the final manuscript.

\section{Availability of data and materials}

The original materials in the report are available from the corresponding author on reasonable request.

\section{Abstract and Manuscript Text word count 1289 words \\ Ethics approval and consent to participate}

The institutional Institutional Review Board (IRB) does not require IRB review/approval for case reports in which three or fewer cases are reported.

\section{Consent for publication}

Consent from the patient was obtained. 


\section{Competing interests}

The authors declare that they have no conflict of interest.

\section{References}

1. Aigmueller T, Dungl A, Hinterholzer S, Geiss I, Riss P (2010) An estimation of the frequency of surgery for posthysterectomy vault prolapse. Int Urogynecol J 21 (3):299-302. doi:10.1007/s00192-009$1033-4$

2. Marcickiewicz J, Kjollesdal M, Engh ME, Eklind S, Axen C, Brannstrom M, Stjerndahl JH (2007) Vaginal sacrospinous colpopexy and laparoscopic sacral colpopexy for vaginal vault prolapse. Acta Obstet Gynecol Scand 86 (6):733-738. doi:10.1080/00016340701332811

3. Florian-Rodriguez ME, Hamner JJ, Corton MM (2017) First sacral nerve and anterior longitudinal ligament anatomy: clinical applications during sacrocolpopexy. Am J Obstet Gynecol 217 (5):607 e601-607 e604. doi:10.1016/j.ajog.2017.07.008

4. Wang YQ, Yang X, Wang JL (2016) [Treatment and outcome of polypropylene mesh or tape related pain after reconstructive pelvic surgery]. Zhonghua Fu Chan Ke Za Zhi 51 (12):901-908. doi:10.3760/cma.j.issn.0529-567X.2016.12.005

5. Vargas R, Keryan A, Minassian VA (2015) Appendiceal adhesion to synthetic mesh after laparoscopic sacrocolpopexy: a case report. Int Urogynecol J 26 (1):155-157. doi:10.1007/s00192-014-2466-y

6. Siddharth A, Cartwright R, Jackson S, Price N (2020) Laparoscopic complete sacrocolpopexy mesh removal for right-sided gluteal pain and recurrent mesh erosion. Int Urogynecol J 31 (2):411-413. doi:10.1007/s00192-019-04088-4

7. Dwyer PL (2011) Neurophysiology in urogynaecology. Int Urogynecol J 22 (12):1471-1472. doi:10.1007/s00192-011-1570-5

8. Seracchioli R, Raimondo D, Arena A, Gava G, Parmeggiani C, Martelli V, Moro E, Zanello M, Paradisi R, Mabrouk M (2018) Laparoscopic Mesh-Less Cervicosacropexy for Uterovaginal Prolapse. Female Pelvic Med Reconstr Surg 24 (6):399-403. doi:10.1097/SPV.0000000000000464

\section{Figures}




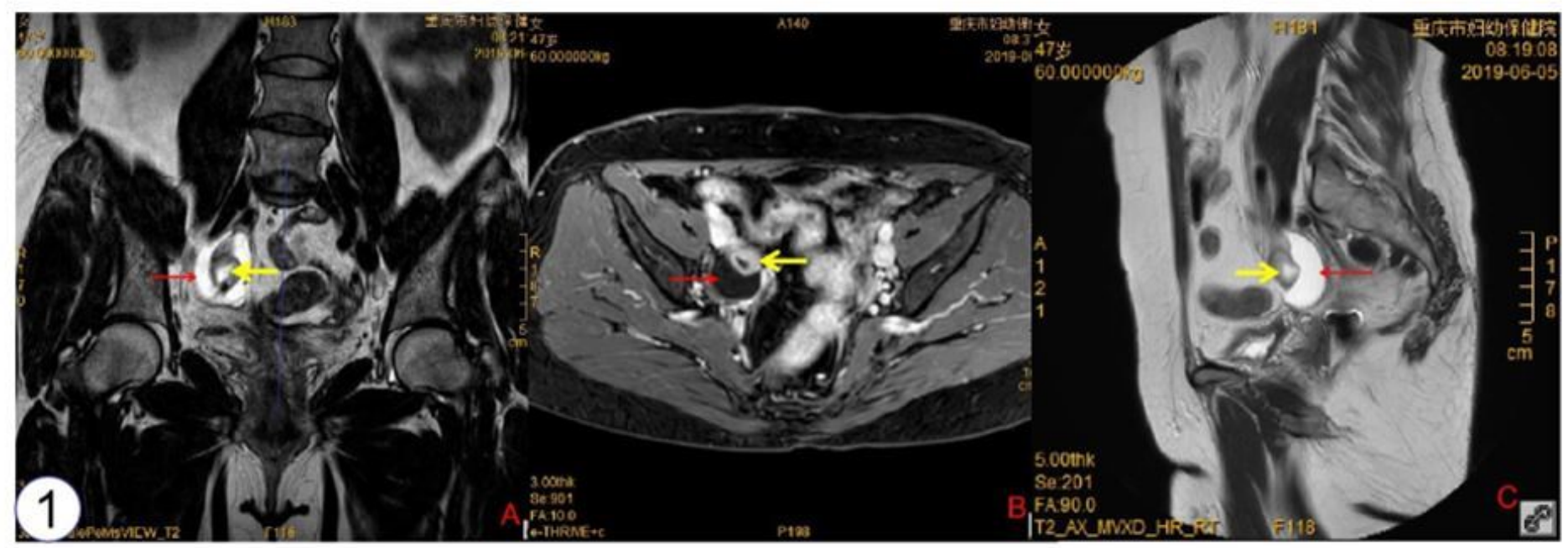

\section{Figure 1}

The mesh (yellow arrow) and encapsulated pelvic effusion (red arrow) are evident in the magnetic resonance images. It is difficult to observe the fixation site on the anterior surface of the first sacral vertebra in magnetic resonance images. (A) Coronal image (B) Transverse image (C) Sagittal image

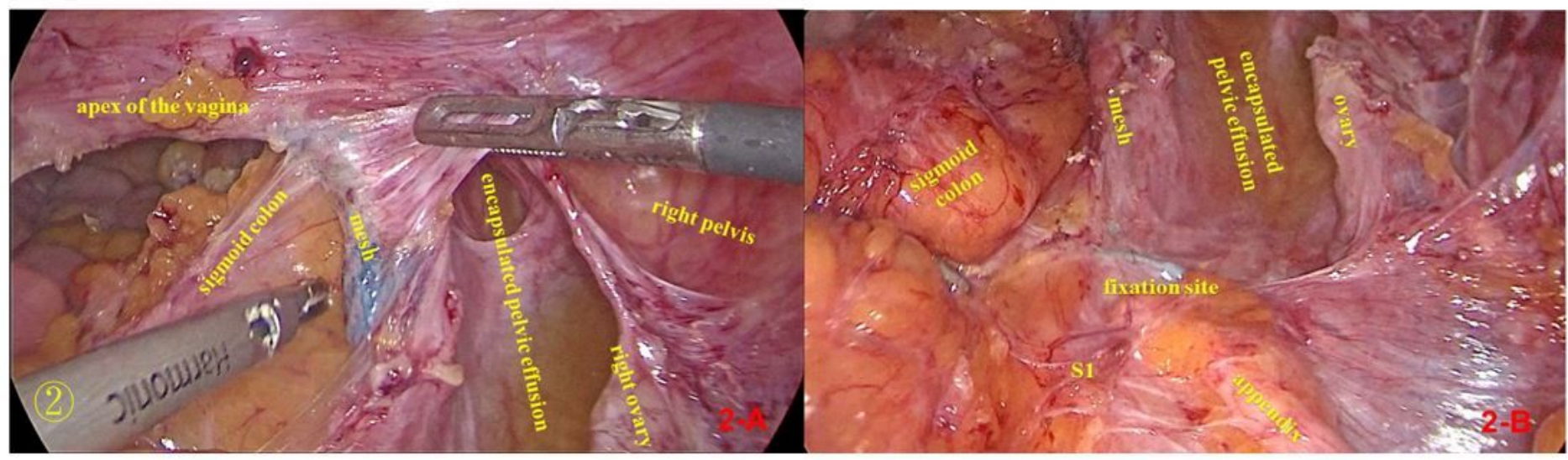

Figure 2

(A) The relationship between the mesh, encapsulated effusion and intestinal canal, which is consistent with the magnetic resonance imaging findings. (B) The relationship between reticulum and surrounding tissues, such as presacral area and encapsulated effusion. 

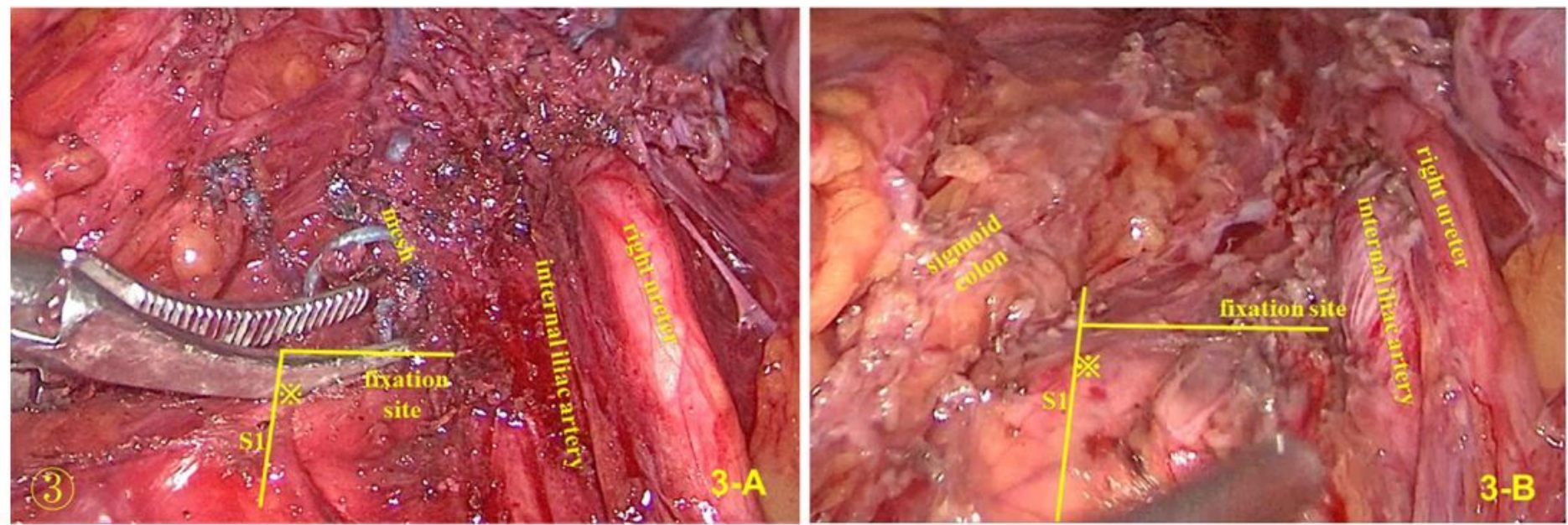

Figure 3

The relationship between the fixation site of the mesh and the surrounding organs, such as the ureter and blood vessel. The fixation site of the intraoperative mesh and the actual position (asterisk) at which fixation should have occurred, demonstrating an obvious deviation. (A) No mesh was removed. (B) After the mesh was removed. 